\title{
Data Transmission using Visible Light Communication
}

\author{
Anoop Kiran A., Seshachalam D.
}

\begin{abstract}
The demand placed on wireless technology which uses Radio Frequency (RF) in the present society is growing exponentially. In the near future, due to the limited bandwidth of $R F$ spectrum, these resources will reach an overload point. $A$ technique of contradicting the radio frequency spectrum issue is the use of Visible Light Spectrum. Light-Fidelity (Li-Fi) is a research field which uses the visible light band within the Visible Light Communication (VLC). This paper describes the research about $V L C$, the design method and testing of a VLC prototype using UART (Universal Asynchronous Receiver/Transmitter) module. The design method consists of serial communication module written in python called pySerial to convert the file data to stream of bits to be sent via LED. The designed prototype is simple, cheap and provides a concrete base for fellow researchers to work further in this field. The principle of VLC is effectively exhibited in this prototype. Also, the feasibility of writing the codes in Python language, proper electronic components used to process bit wise data signals and advantages of serial communication for data transfer are successfully displayed. VLC has major application in indoor communication, underwater communication and in hospitals where radio waves causes Electromagnetic Interference.
\end{abstract}

Keywords: Bit Error Ratio (BER), Graphical User Interface (GUI), Li-Fi, Transimpedance Amplifier (TIA), UART.

\section{INTRODUCTION}

A method of telecommunication which uses light as the transmission medium between two devices is called optical communication. It originated as beacon fires and smoke signals that deliver a message in prehistoric times, optical wireless communication (OWC) has advanced to a high-capacity complementary technology to radio frequency communication. Optical Wireless Communication systems use wavelengths in visible light spectrum for Visible Light Communication (VLC) and the infrared spectrum for IR communication. The availability of Visible light spectrum is huge (10,000 times larger than RF band) and it has the prospective to deliver wireless links with high data rates. A massive amount of concurrent data can be carried by the

Revised Manuscript Received on February 05, 2020.

* Correspondence Author

Anoop Kiran A*, M Tech-Electronics, Department of Electronics and Communication Engineering, BMS College of Engineering, Bengaluru, India. Email: anoopkiran.lel17@bmsce.ac.in

Dr. Seshachalam D., Department of Electronics and Communication Engineering, BMS College of Engineering, Bengaluru, India. Email: dschalam.ece@bmsce.ac.in

(C) The Authors. Published by Blue Eyes Intelligence Engineering and Sciences Publication (BEIESP). This is an open access article under the CC BY-NC-ND license (http://creativecommons.org/licenses/by-nc-nd/4.0/) modulated Light waves of VLC, at greater speeds, which cannot be handled by current consumer equipment. [1].

Dr Harald Hass was the first researcher to introduce LI-Fi to the world in his TED talk in 2011. He is currently the leading worldwide exponent in this field and has his own company called pureLiFi. Since 2011, there has been exponential rise in the field of LI-Fi research.

A comprehensive research was published by Kalavally where they run through each marketable LED type and describe their properties. They also differentiate and describe the best suitable photodiode (PD) between an Avalanche photodiode and PIN photodiode [2].

The prototype implemented by Nakul Garg between two devices such as mobiles and computers make use of UART modules with LASER for an extended range and concentrated brightness. Their results show that any format of data can be transmitted regardless of the surrounding light sources with data rate of 300 bits per second (bps) at a distance of 2 meters [3].

A portable device prototype is made by Shreyash A. Pande that establishes VLC communication between two or more computers. They have used UART for serial communication and have programmed a GUI in MATLAB. They have transmitted a string of characters at a speed of 93 Bytes per second (Bps) over a distance of $90 \mathrm{~cm}$ [4].

A LiFi prototype designed by Mohit Vasuja consists of an IR LED transmitter, photodiode and a PIC microcontroller which works on the UART serial communication protocol. They have successfully transmitted text and image with a speed of 75 Bytes per second (Bps) [5].

The prototype explained in this paper consists of:

\section{LED Transmitter}

The LEDs required for a high speed data communication system need have modulation rate of at least $2-3 \mathrm{MHz}$ to ensure the perceptible rate of human eye. A $1 \mathrm{~W}$ high power white LED was used in the design of this prototype because of its wide wavelength bandwidth that is detectable by most of the photodiodes [6].

\section{Photodiode Receiver}

Photodiodes can be connected in two different modes; photovoltaic and photoconductive. Given that the circuit operates in low power and low current configuration, photovoltaic mode is selected and Vishay Semiconductors' BPW34 photodiode was selected considering low capacitance of the PD and the cost [7]. 


\section{Data Transmission using Visible Light Communication}

\section{Transimpedance Amplifier}

In order to receive the low voltage from the photodiode, OPA380 transimpedance amplifier was used to convert the low current of the PD to high voltage.

\section{UART}

An UART module was required for asynchronous serial communication to send the bit data to LED. UARTs consist of a shift register used to convert serial data to parallel or vice-versa.

\section{METHODOLOGY}

The design of the prototype is broken in to two modules; Transmitter (Tx) module and Receiver (Rx) module. Fig. 1 shows the prototype system diagram: each module consists of the software subsystem and the hardware subsystem (External Circuit) which is explained below.

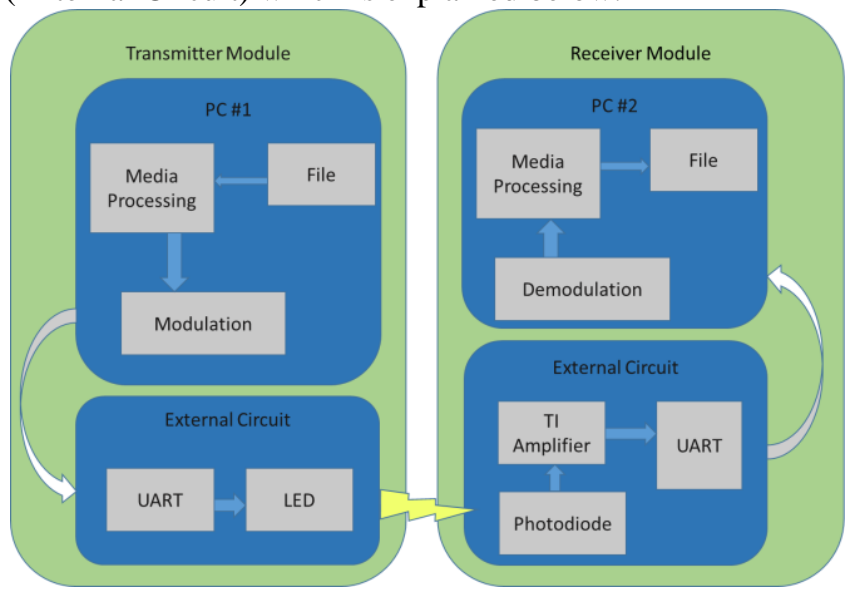

Fig. 1. Prototype System Diagram

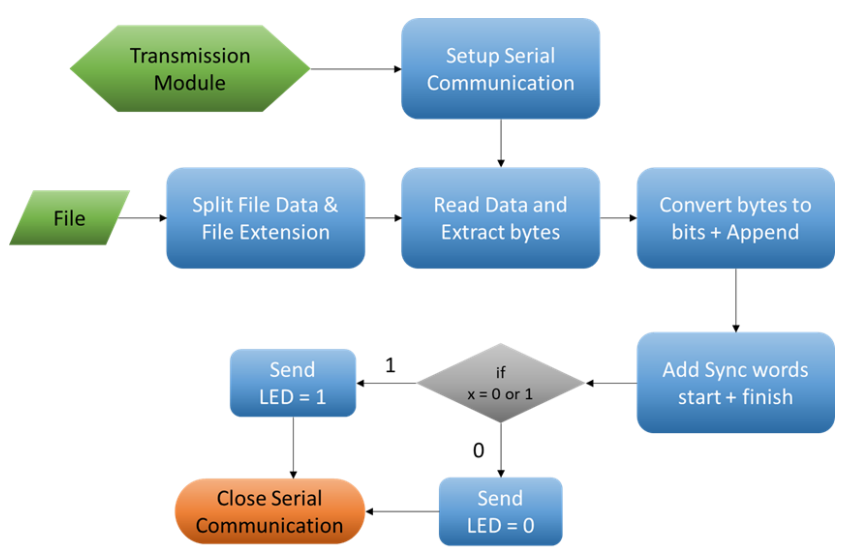

Fig. 2. Software Diagram for Transmission Module

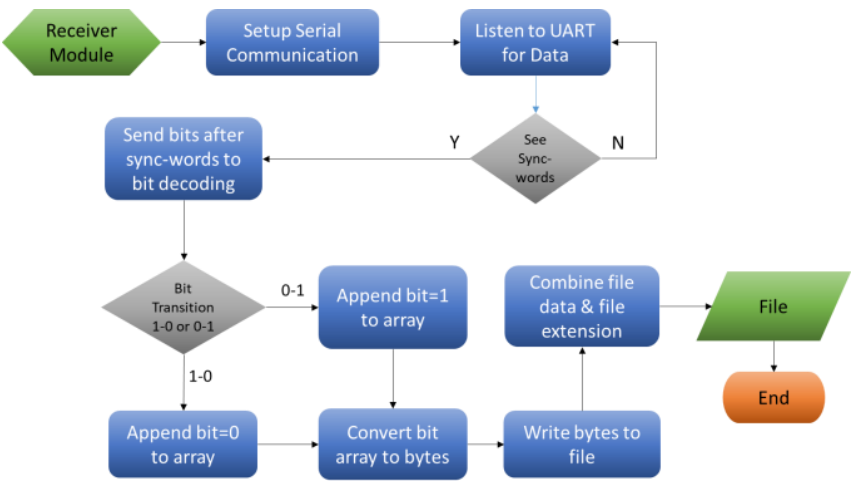

Fig. 3. Software Diagram for Receiver Module

\section{A.Software Subsystem:}

The Transmitter module has the function of file selection, binary data extraction, coding to serial data, processing that data through an UART and LED flickering. Fig. 2 shows the software diagram of the transmitter module.The code was written in python and sync-words were added to identify the start and end of the data file. Serial communication was used to transmit the file data from LED by using pySerial library in python. The Receiver module had a similar configuration of reverting the received bits from the photodiode, processing the serial bit data through an UART, combining the bits to form original file data and finally saving it to the file. Fig. 3 shows the software diagram of the receiver module.

The main coding point of the receiver circuit was to keep checking the Rx pin of UART, looking for the sync words. Once they were recognized, the entire bits were received.

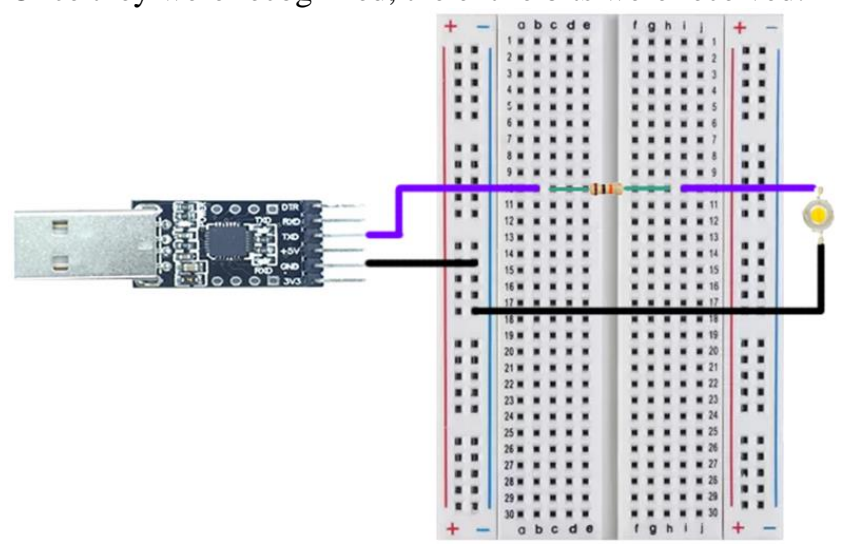

Fig. 4. Transmitter Module Layout Diagram

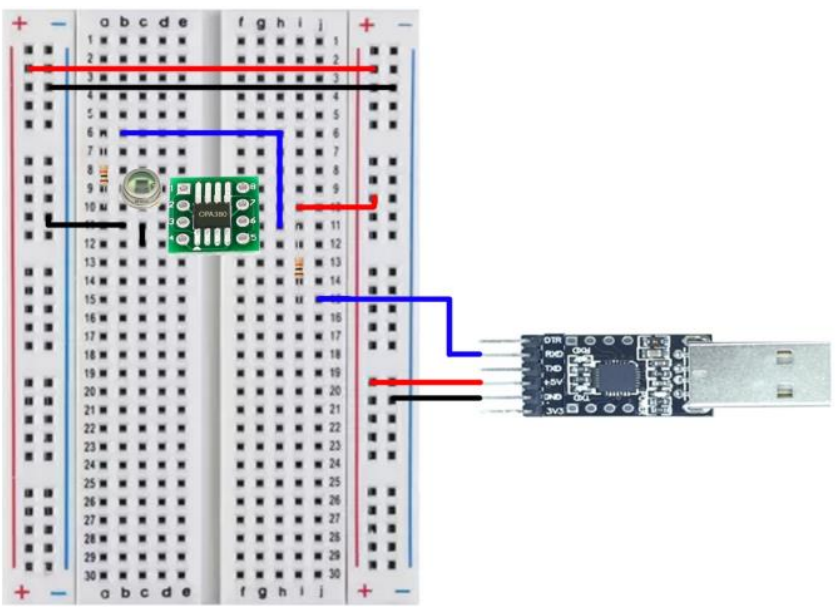

Fig. 5. Receiver Module Layout Diagram

\section{B.Hardware Subsystem}

In building a hardware circuit, the transmitter consisted of a USB-TTL UART, $1 \mathrm{~W}$ LED and a $330 \Omega$ resistor shown in fig.4. Dim light was produced by the $1 \mathrm{~W}$ LED, so the $330 \Omega$ resistor was removed and tested which resulted in increased intensity of the LED light. Also, a reflector was added the LED to increase the directivity of the light onto the photodiode. The receiver circuit needed more components because the voltage received from the photodiode was too low and could not be recognized by the UART.

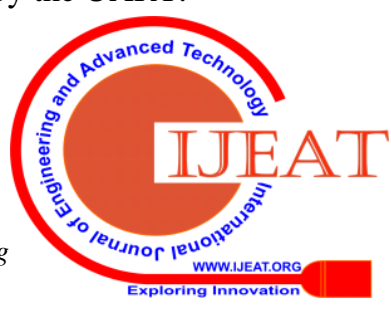


Hence a transimpedence amplifier (TIA) was used to convert the low

current to high voltage of the range $2 \mathrm{~V}$ to $3.3 \mathrm{~V}$ which was recognized by the UART.

Fig. 5 shows the receiver circuit in which OPA380 is used as a TIA, cathode of the BPW34 Photodiode connected to Pin 2 of the TIA and anode grounded, $330 \Omega$ resistor connected between Pin 6 of TIA and Rx Pin of UART, $1 \mathrm{M} \Omega$ resistor connected between Pins 2 and 6 of TIA, pins 3 and 4 of the TIA are grounded.

\section{C.Prototype Module Integration}

When both the transmitter and receiver modules were finalized and tested individually, they were kept in line-of-sight to each other and data was sent at various distances with different baud rates. Fig. 6 shows the test environment of the prototype.

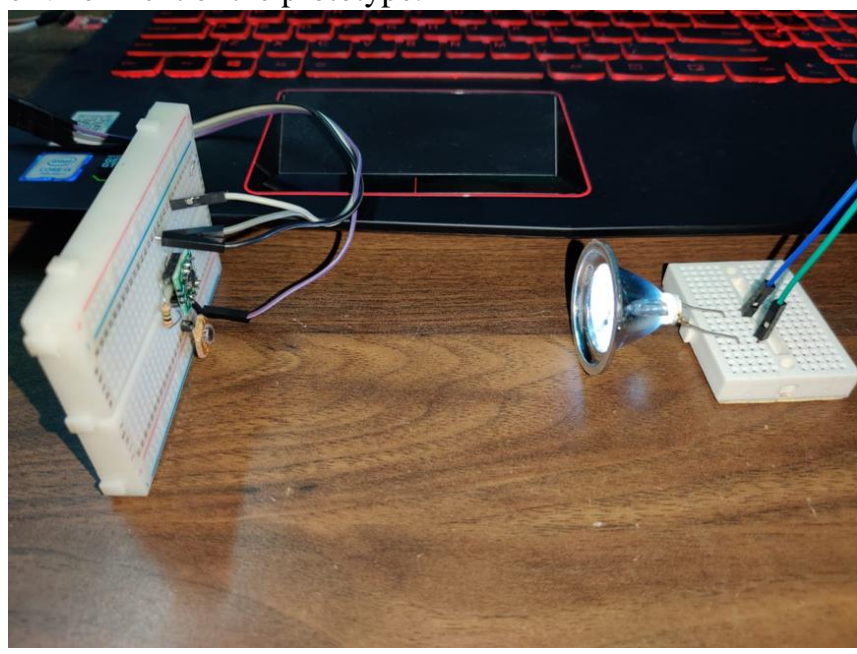

Fig. 6. Prototype Test Environment

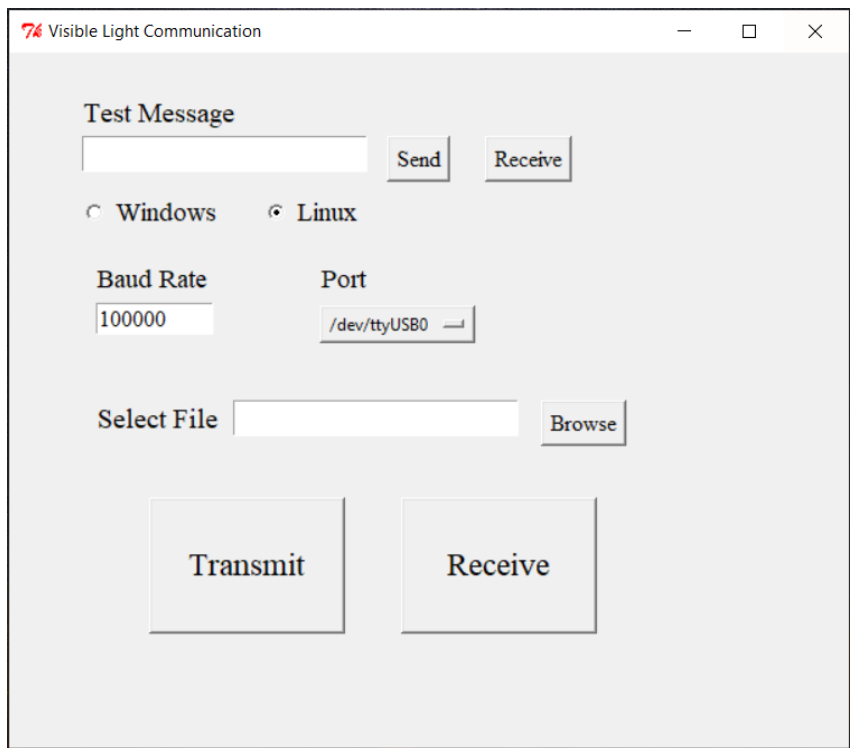

Fig. 7. GUI of Visible Light Communication

\section{RESULT AND DISCUSSION}

Before the processing of file data, the prototype was tested with an infinite loop of letters sent from the transmission module. The receiver was placed in line-of-sight and adjusted till the proper letters were received. When the codes are executed simultaneously, the output can be seen as 'Hello Everyone' being shown infinitely.
Then the prototype was tested for various file types (.jpg, .mp4, .mp3, .txt) of data transmission individually and then they were merged together in a single program. Also, GUI (Graphical User Interface) was written and executed for the ease of project. Fig. 7 shows the GUI developed for this project.

The whole execution part of the VLC GUI included a test message which was used to verify the correctness of the transmitted data as it was seen on the receiving side. It has an option of selecting the OS which is being used for the project. The Ports of the transmitting UART and receiving UART can also be selected from the GUI.

The default baudrate is set to $100 \mathrm{k}$, which can be changed according to the user. Finally, any type of file can be browsed and sent from the transmitting module to the receiver module by first clicking 'Receive' on the receiver GUI and then clicking 'Transmit' on the transmitter GUI within 2 Seconds, which is the timeout period.

The speed analysis of this prototype was done by varying values of baudrate shown in fig. 8. The transmission speed reaches up to 25 kilobytes per second (kBps) with no errors and then after has few errors which is shown in the BER analysis.

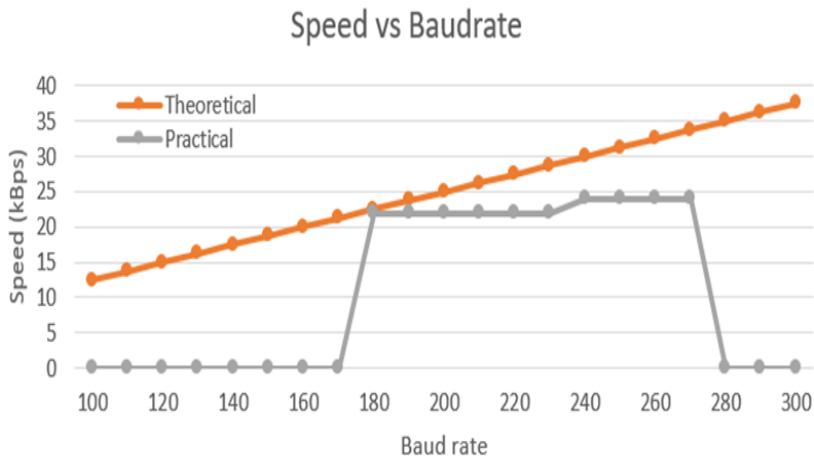

Fig. 8. Speed in kBps vs Baud rate of the VLC prototype BER (\%) vs Distance $(\mathrm{cm})$

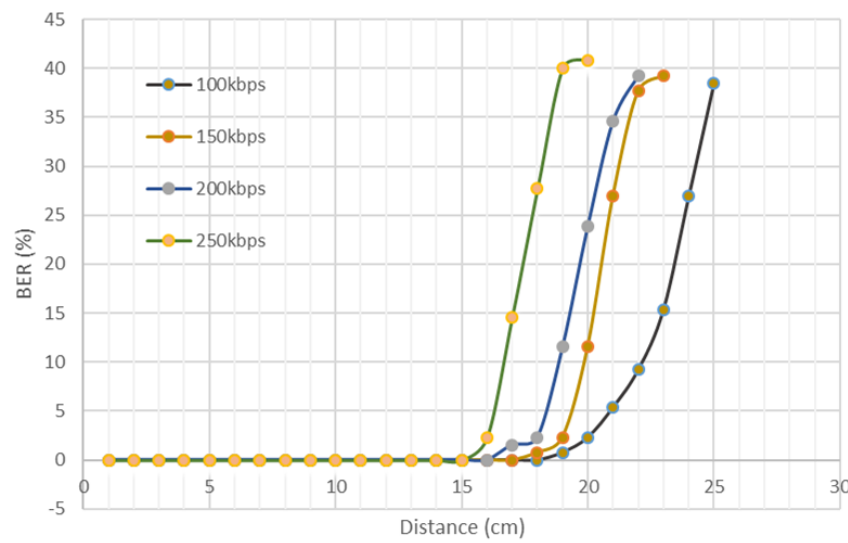

Fig. 9. BER Analysis at various transmission speeds

The BER (Bit Error Ratio) analysis was done at various distances between LED and Photodiode at different transmission speeds by transmitting a sequence of data and converting the received data to binary and checking the number of error bits. The Bit Error Ratio is calculated using the formula:

Published By:

Retrieval Number: C5130029320/2020@BEIESP 


\section{Data Transmission using Visible Light Communication}

BER $(\%)=($ No of error Bits / Total number of transmitted bits) $\times 100$

The BER analysis shows the error percentage rising as the distance is increased. This is because of the noise interfering with transmission. Also, the BER percentage varies for different speeds as shown in fig. 9. At higher speeds, the error in transmission and reception of bits is increased compared to lower speeds at the specified distance.

\section{CONCLUSION AND FUTURE SCOPE}

The working model of a VLC prototype has been implemented successfully between two personal laptops. The prototype designed here is capable of transmitting any type of data by reading the file in text format and reverting it back to original file in the receiver end using serial communication. The system achieves a distance up to 15 centimeters with no errors at a speed of $25 \mathrm{kBps}$. The flickering of LED maybe interfered with noise when tested in daylight and produces errors but operates well in dim lit areas.

The price of the prototype was considerably low and further, by increasing the physical dimensions of the transmitter and receiver and by using high grade components, the prototype can be made more efficient while achieving greater speeds and longer distances. While this prototype consists of both the transmitter and receiver modules, in the future they can be combined to make one transceiver.

If this technology is put into practical use, then every bulb can be used as a Li-Fi module to transmit and receive the data at the same time. The benefit of not using the RF communication is such that Li-Fi technology can be used in airplanes, underground mines, submarines and other places where Radio Frequency waves are not desired [8]. Speed of the communication could not be increased because of the limitations of UART serial communication and also the use of low power LED, thus it was not possible to transmit large data files. Better processors can be used to overcome these limitations. Also interference of sunlight and other lights is a big challenge.

\section{ACKNOWLEDGMENT}

My deep sense of gratitude to my guide Dr. D. Seshachalam, Professor, Department of Electronics and communication Engineering, BMS College of Engineering, Bengaluru, for his valuable time and guidance and precious suggestions regarding the project.

\section{REFERENCES}

1. Paul Fergusson, "Light Fidelity (Li-Fi) Prototype with Raspberry Pi", University of Southern Queensland, Faculty of Health, Engineering and Sciences, October 2016.

2. Kalavally V, Zafar F, Kalavally Karunatilaka D V \& Parthiban R, "LED Based Indoor Visible Light Communications: State of the Art", Communications Surveys \& Tutorials, IEEE, vol.17, no.3, pp.1649-1678, 2015.

3. Nakul Garg, Jolly Parikh, "Wireless transceiver design for visible light communication", 9th International Conference on Computing, Communication and Networking Technologies (ICCCNT), 2018.

4. Shreyash A. Pande, Shruti J. Sonar, Shubham K. Ingale, "Optical data transmission using portable USB Li-Fi module (dongle)", International Conference on Computer Communication and Informatics (ICCCI), 2018.

5. Mohit Vasuja, Amritansh Kumar Mishra, Udbhav Singh Chauhan, Deeksha Chandola, Shashank Kapoor, "Image Transmission Using
Li-Fi", Second International Conference on Inventive Communication and Computational Technologies (ICICCT), 2018.

6. Ruonan Ji, Shaowei Wang, Qingquan Liu and Wei Lu, "High-Speed Visible Light Communications: Enabling Technologies and State of the Art", Applied Sciences, MDPI, April 2018.

7. https://en.wikipedia.org/wiki/Photodiode .

8. Anurag Sarkar, Shalabh Agarwal and Asoke Nath, "Li-Fi Technology: Data Transmission through Visible Light", International Journal of Advance Research in Computer Science and Management Studies, July 2015.

\section{AUTHORS PROFILE}

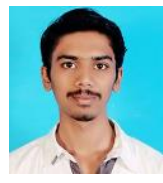

A Anoop Kiran is pursuing his Master's Degree in Electronics in the department of Electronics and Communication Engineering at B.M.S. College of Engineering, Bengaluru, India. He has secured his Bachelor Degree in Electrical and Electronics Engineering from M.S.Ramaiah Institute of Technology. His field of research interests are Optical telecommunication and Embedded Systems specifically Robotics and Internet of Things.

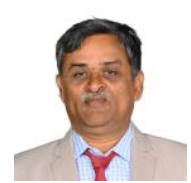

Dr. D Seshachalam is a professor and former HoD in the department of Electronics and Communication Engineering, B.M.S. College of Engineering, Karnataka, India. His field of research interests are Model order reduction power electronics non-linear control engineering battery management systems in EHV. To his credit: guiding research scholars, authored or co-authored, presented and published number of research papers in reputed conferences and Journals. He has been a member of various academic forums at Visvesvaraya Technological University, Belgaum, India. 